Cite this: Phys. Chem. Chem. Phys., 2014, 16, 16524

Received 21st March 2014 , Accepted 20th June 2014

DOI: $10.1039 / c 4 c p 01234 h$

www.rsc.org/pccp

\section{Molecular motors pulling cargos in the viscoelastic cytosol: how power strokes beat subdiffusion}

\author{
Igor Goychuk, ${ }^{\star a}$ Vasyl O. Kharchenko ${ }^{\mathrm{b}}$ and Ralf Metzler ${ }^{\mathrm{ac}}$
}

\begin{abstract}
The discovery of anomalous diffusion of larger biopolymers and submicron tracers such as endogenous granules, organelles, or virus capsids in living cells, attributed to the viscoelastic nature of the cytoplasm, provokes the question whether this complex environment equally impacts the active intracellular transport of submicron cargos by molecular motors such as kinesins: does the passive anomalous diffusion of free cargo always imply its anomalously slow active transport by motors, the mean transport distance along microtubule growing sublinearly rather than linearly in time? Here we analyze this question within the widely used two-state Brownian ratchet model of kinesin motors based on the continuous-state diffusion along microtubules driven by a flashing binding potential, where the cargo particle is elastically attached to the motor. Depending on the cargo size, the loading force, the amplitude of the binding potential, the turnover frequency of the molecular motor enzyme, and the linker stiffness we demonstrate that the motor transport may turn out either normal or anomalous, as indeed measured experimentally. We show how a highly efficient normal active transport mediated by motors may emerge despite the passive anomalous diffusion of the cargo, and study the intricate effects of the elastic linker. Under different, well specified conditions the microtubule-based motor transport becomes anomalously slow and thus significantly less efficient.
\end{abstract}

\section{Introduction}

The thermally driven Brownian motion represents a ubiquitous transport mechanism in aqueous solution, especially for small particles such as metal and salt ions, aminoacids, sugar molecules, and even for larger particles such as transcription factors, enzymes, lipid granules, or RNAs (on scales larger than their gyration radius). The mean squared displacement (MSD) covered by a Brownian particle without external bias $(\langle\mathbf{r}\rangle=0)$ scales linearly with time, $\left\langle(\delta \mathbf{r})^{2}\right\rangle=\left\langle\mathbf{r}^{2}\right\rangle-\langle\mathbf{r}\rangle^{2} \propto D t$. If an external force $\mathbf{f}_{\mathrm{ext}}$ acts on the particle, the mean distance in the direction of the bias also scales linearly with time, $\langle\mathbf{r}\rangle \propto \mathbf{f}_{\text {ext }} t \eta \eta$. The diffusion coefficient $D$, the viscous friction coefficient $\eta$, and the temperature $T$ are then related by the Einstein-Stokes relation $D=k_{\mathrm{B}} T / \eta$, where $k_{\mathrm{B}}$ is the Boltzmann constant. This relation manifests the classical fluctuation-dissipation theorem (FDT) at local thermal equilibrium. ${ }^{1}$ The Stokes formula $\eta=6 \pi \zeta a$ relates the viscous friction with the viscosity $\zeta$ of the medium and the radius $a$ for

\footnotetext{
${ }^{a}$ Institute for Physics and Astronomy, University of Potsdam, Karl-Liebknecht-Str. 24/25, 14476 Potsdam-Golm, Germany. E-mail: igoychuk@uni-potsdam.de; Fax: +49-331-977-1045; Tel: +49-331-977-5614

${ }^{b}$ Institute of Applied Physics, Natl. Acad. Sci. Ukraine, Sumy, Ukraine

${ }^{c}$ Department of Physics, Tampere University of Technology, Korkeakoulunkatu 3, 33101 Tampere, Finland
}

spherical particles in simple fluids. The mobility $\mu=1 / \eta$ and diffusion coefficient of a particle are thus inversely proportional to its size.

Molecular transport inside living biological cells comprises both passive and active motion. ${ }^{2}$ In complex molecularly crowded polymeric fluids such as the cellular cytosol, ${ }^{3}$ the above simple linear dependence of the friction coefficient on the particle size generally breaks down, and the effective friction coefficient $\eta_{\text {eff }}$ may even be exponentially enhanced in the liquid, with respect to the one in water by many orders of magnitude. This effect depends on the particle size, the correlation length of the polymeric fluid, and other parameters. ${ }^{4,5}$ For instance, if for a calcium ion the viscosity in the cytosol of a cell is essentially the same as in water, a vesicle or magnetosome with radius $a>100 \mathrm{~nm}$ may rather experience an effective viscosity comparable to the one of glycerol which is some $1500 \times$ more viscous than the one of water at room temperature, or even honey $(10000 \times$ more viscous), as extrapolated from recent systematic studies in ref. 6 for a more simple system. Even for particles of a typical size of a globular protein $(a \sim 2.5 \mathrm{~nm})$ the enhancement factor can be as large as 750 , as derived in ref. 7 from the experimental data in ref. 8.

Indeed, the passive diffusion of submicron particles in living cells slows down tremendously, if to consider it as a normal 
diffusion process on the macroscale, with respect to the one in pure water. However, a more dramatic effect is that the cytosol actually behaves as a viscoelastic polymeric fluid and displays profound memory effects. Viscoelasticity can cause anomalous diffusion in the subdiffusive range, displayed by the sublinear scaling of the MSD, $\left\langle(\delta \mathbf{r})^{2}\right\rangle \propto D_{\alpha} t^{\alpha}$ with $0<\alpha<1$, where the anomalous diffusion coefficient is $D_{\alpha}$. Such subdiffusion was established in numerous experiments based on a large range of tracer particles both in the cytosol of living cells and in controlled crowded environments in vitro. ${ }^{8-26}$ Although subdiffusion mechanisms other than viscoelasticity also appear relevant to explain experimental results, such as continuous time random walk-type motion, ${ }^{19,21-23}$ and these different types of anomalous diffusion may even mix in some systems, ${ }^{19,22}$ on time scales relevant to our study the viscoelastic nature of the subdiffusion appears to be well established both in vivo and in control experiments in vitro. ${ }^{16,19-23,25-27}$

Viscoelastic subdiffusion is related to the famed fractional Brownian motion, ${ }^{28,29}$ as discussed in ref. 30 . This connection can be derived ${ }^{31,32}$ from a Generalized Langevin Equation (GLE) $)^{1,33}$ with a power-law memory kernel and fractional Gaussian thermal noise, ${ }^{30,34}$ see below. Dynamically the GLE governed motion with power-law correlations represents a well-founded approach with deep roots in statistical mechanics. ${ }^{33,35,36}$ As shown in the above experiments, the highly crowded cytosol ${ }^{37,38}$ is such a viscoelastic environment, and we are thus going to use the GLE approach with power-law correlations in the present work. As a guiding example, we consider the subdiffusive motion of magnetosomes with submicron radius $a=300 \mathrm{~nm}$ and chains thereof consisting of up to 8 magnetosomes investigated experimentally in ref. 17 . These magnetosomes perform passive subdiffusion with anomalous diffusion exponent $\alpha \approx 0.4$ in the intact cytosol. Their motion is characterized by subdiffusion coefficients as small as $D_{0.4} \sim$ $10^{-16} \mathrm{~m}^{2} \mathrm{~s}^{-0.4}=100 \mathrm{~nm}^{2} \mathrm{~s}^{-0.4}$ and even smaller, depending on the number of magnetosomes in the chain. Therefore, the corresponding subdiffusion spreading within one second is of the order of merely $10 \mathrm{~nm}$.

Without help of active transport by molecular motors such particles would be practically localized on even appreciably long time scales. Pulling by molecular motors under consumption of energy provided by ATP is thus crucial for the delivery of such and similar cargos to more distant locations in living cells. $^{39,40}$ Given the coupling of such cargos to the viscoelastic properties of their environment in the cytosol and the resulting passive subdiffusion of cargos, the immediate question arises: how does this affect the active transport by molecular motors? This is the focus of the present work.

The theory of molecular motors viewed as Brownian stochastic engines is well developed for Markovian dynamics in absence of any memory effects. ${ }^{41-48}$ Generalizing these models systematically to the motor motion in viscoelastic media was started to be studied in ref. 34 and $49-53$ by extending several popular models of Brownian ratchets ${ }^{48}$ towards the dynamics with long-range viscoelastic memory. Such ratchets are indeed closely related to molecular motors. ${ }^{41-45,48,54}$ Within the framework of such ratchet models one can think of the motion of a compound, tightly coupled motor-cargo particle as one moving in a periodic external force field provided by interaction with the polymeric fibers of the cytoskeleton, e.g., the microtubules in the case of kinesin motors considered here. A generalization of such a standard continuous diffusion ratchet model of molecular motors to include viscoelastic subdiffusion has been put forward recently. ${ }^{55}$ This model explains a number of experimental facts, in particular, that the transport by molecular motors in the viscoelastic cytosol may be both normal and anomalously slow, depending on physical parameters such as the motor's turnover frequency and the cargo size.

In the present work, to become more realistic we establish a further generalization by considering the motor-mediated transport of large subdiffusive cargos, that are attached to the motor by elastic linkers. That is, we consider motor and cargo as two independent particles and thus relax the assumption of the absolutely rigidly linked motor-cargo compound. Similar elastic linker models were considered for the normal diffusion of both cargo and motor in dilute solvent conditions. ${ }^{56}$ In our approach we assume that the passive diffusion of the motor is normal, without memory effects, when it is not attached to a cargo particle. We characterize the fact that the motor moves in the viscoelastic cytosol and not just water by a significantly reduced (by a factor of 10) diffusion coefficient with respect to the one expected in water. This assumption is justified by the relatively small size of the motor protein itself. However, the coupling to the subdiffusive, comparatively large cargo enforces the subdiffusion of the coupled motor-cargo unit, as long as it is not coupled to a microtubule. Considering experimentally relevant elastic constants of the motor-cargo linker from ref. 18, 57 and other realistic motor parameters we confirm the coexistence of normal and anomalous motor transport for certain parameter values similar to the findings of ref. 55. However, as we discuss here, in this more general setup of an elastic coupling between motor and cargo, the anomalous transport regime becomes in fact reinforced. It is shown to emerge already for motor turnover frequencies of the order of $100 \mathrm{~Hz}$ for sufficiently large cargos of a typical radius $a=300 \mathrm{~nm}$. However, even for such large cargo particles the transport can become normal if the turnover frequency is lowered to $10 \mathrm{~Hz}$. Generally, the dependence of the effective subdiffusive transport exponent on the cargo size and the motor turnover frequency can provide a decisive experimental support for anomalous motor transport in living cells.

\section{Theory and modeling}

Diffusion in complex viscoelastic fluids such as the cytosol is commonly described by the GLE. ${ }^{58,59}$ We here consider overdamped dynamics of particles, neglecting inertial effects, as appropriate for the low Reynolds number limit for submicron particles in the cytosol. We use the single Cartesian coordinate $y$ for such a particle. It corresponds to the chemical coordinate of the motion along the microtubule when the particle becomes 
attached to the motor moving on the microtubule. The GLE then reads

$$
\int_{-\infty}^{t} \eta_{\mathrm{c}}\left(t-t^{\prime}\right) \dot{y}\left(t^{\prime}\right) \mathrm{d} t^{\prime}=f_{\text {ext }}(t)+\xi_{\mathrm{c}}(t) .
$$

Here the memory kernel $\eta_{\mathrm{c}}(t)$ and the autocorrelation function of unbiased, thermal colored Gaussian noise $\xi_{\mathrm{c}}(t)$ are related by the second FDT of Kubo,

$$
\left\langle\xi_{\mathrm{c}}(t) \xi_{\mathrm{c}}\left(t^{\prime}\right)\right\rangle=k_{\mathrm{B}} T \eta_{\mathrm{c}}\left(\left|t-t^{\prime}\right|\right)
$$

named also the fluctuation-dissipation relation. It reflects the balance at thermal equilibrium between the energy supplied by thermal noise and the energy dissipated due to friction. The stochastic description in terms of the GLE (1) is not only consistent with the laws of equilibrium statistical physics and thermodynamics, but it also allows one to treat strongly outof-equilibrium transport driven, e.g., by a non-thermal fluctuating force $f_{\text {ext }}(t)$. The GLE (1) serves as a basis in passive microrheology, ${ }^{11,58,59}$ at thermal equilibrium, to derive the complex shear modulus of the medium in the frequency domain, $G^{*}(\omega) \propto i \omega \int_{0}^{\infty} \exp (-i \omega t) \eta(t) \mathrm{d} t$, from measured particle trajectories.

The frequency dependence of the complex shear modulus is commonly used to characterize viscoelastic materials..$^{8,11,12,58-60}$ In particular, the frequently observed power-law scaling $G^{*}(\omega) \propto$ $(i \omega)^{\alpha}$ with $0<\alpha<1$ corresponds to subdiffusion of a free particle in this medium, $\left\langle(\delta x)^{2}\right\rangle \propto D_{\alpha} t^{\alpha}$, with fractional diffusion coefficient $D_{\alpha}$ and power-law memory kernel, $\eta(t) \propto \eta_{\alpha} / t^{\alpha}$, where $\eta_{\alpha}$ is the fractional friction coefficient obeying the fractional Einstein relation $D_{\alpha}=k_{\mathrm{B}} T / \eta_{\alpha} \cdot{ }^{34}$ In the macroscopic theory of viscoelasticity, similar memory kernels were introduced by A. Gemant ${ }^{61}$ as a generalization of the simplest Maxwell model with exponential memory decay. ${ }^{62}$ In practice, the power-law scaling extends over a finite number of time and frequency decades. A high-frequency (short-memory) cutoff reflects the molecular nature of the condensed medium. A low-frequency, or long-memory cutoff guarantees that the macroscopic friction coefficient $\eta_{\text {eff }}=\int_{0}^{\infty} \eta(t) \mathrm{d} t$ remains finite, reflecting the finite viscosity of any fluid on the macroscale. ${ }^{30,34}$ The intermediate power-law scaling gives rise to subdiffusion on a transient time scale of up to several minutes, depending on the particle size, and this can establish subdiffusion as a primary passive transport mechanism for submicron particles on the mesoscale inside biological cells.

The mathematical model of a strictly algebraically decaying memory kernel corresponds to the fractional Gaussian noise (fGn) model of thermal noise. fGn presents a time-derivative of the fractional Brownian motion (fBm). It was introduced by Mandelbrot and van $\mathrm{Ness}^{29}$ who popularized the concept mathematically introduced by Kolmogorov. ${ }^{28}$ Both can be characterized by the Hurst exponent $H^{\prime}=1-\alpha / 2$, where $\alpha$ is the anomalous diffusion exponent defined earlier for the MSD. fGn is termed persistent for $1 / 2<H^{\prime}<1$, i.e., it exhibits positive correlations. It corresponds to the sub-Ohmic model of thermal baths consisting of harmonic oscillators. ${ }^{63}$ The corresponding
GLE can be derived from a purely dynamic hyper-dimensional Hamiltonian model assuming merely initial canonical distribution of thermal bath oscillators at a given temperature, like in a typical molecular dynamics setup. ${ }^{33,63}$ The solution of GLE (1) is then also fBm, but anti-persistent and subdiffusive, with Hurst exponent $H=\alpha / 2$. This transformation occurs due to the friction with algebraically decaying memory: ${ }^{32}$ high noise values correspond to a high friction. Importantly, this friction memory is at the heart of the very phenomenon of viscoelasticity. Experimental values of $\alpha$ vary significantly and are in the range $\alpha=$ $0.2-1 .^{23}$ For example, for the intact cytoskeleton in ref. 17, $\alpha=$ 0.4 . About the same value can be derived from experimental data in ref. 18, namely from the power spectrum $S(\omega)$ of the transversal position fluctuations of the melanosome particles (size $a=$ $250 \mathrm{~nm}$ ), that are elastically attached to the motor proteins walking along the microtubules. For sufficiently large frequencies (exceeding the inverse relaxation time scale in a parabolic potential well), $S(\omega) \propto 1 / \omega^{b}$, with $b=1+\alpha$, and the experiment yields $b=1.41 \pm 0.02$. We use $\alpha=0.4$ as an experimentally relevant numerical value in what follows.

\subsection{Earlier modeling}

The simplest way to model the action of molecular motors driving a cargo within the GLE approach is to approximate their collective action in terms of a time-dependent random force $f_{\text {ext }}(t)$, which itself exhibits a long range memory and is powerlaw correlated, $\left\langle f_{\text {ext }}\left(t^{\prime}\right) f_{\text {ext }}(t)\right\rangle \propto 1 /\left|t-t^{\prime}\right|^{\gamma}$, with $0<\gamma<1$. This can lead to superdiffusion, ${ }^{64}\left\langle\delta y^{2}\right\rangle \propto t^{\beta}$, with $\beta=2 \alpha-\gamma>1,{ }^{31,64}$ for $\alpha>0.5$. Notice, however, that $\beta$ in this approach can only take the maximal value $2 \alpha$ for $\gamma \rightarrow 0$. This corresponds to a strict $1 / f$ noise property of the driving force $f_{\text {ext }}(t)$ generated by motors with almost non-decaying correlations. The origin of this limit can be easily understood when we consider the transport by a time-alternating force $\pm f_{0}$ in opposite directions. Then, $\langle\delta y(t)\rangle \propto$ $\pm f_{0} t^{\alpha}$, for a given force realization, and after averaging over the driving force fluctuations $\left\langle\langle\delta y(t)\rangle^{2}\right\rangle_{f_{0}} \propto t^{2 \alpha}$. Any decaying correlations of the alternating driving force render the effective diffusion exponent smaller than $\beta_{\max }=2 \alpha$. Clearly, within such a description superdiffusion caused by the activity of molecular motors could only be possible for $\alpha>0.5$, at odds with experimental results showing $\beta \sim 1.2-1.4$ for $\alpha=0.4 .{ }^{17}$ Similarly, the experimental results of ref. 65 cannot be explained within such a model, either: as shown in Fig. 3 in that reference, the passive subdiffusion of lipid droplets with $\alpha \approx 0.58$ is changed to superdiffusion with $\beta \approx 1.74$ when they are actively transported by molecular motors. Compared with experimental evidence, the approach in terms of a time-dependent random force is therefore too simple although it provides a number of useful insights.

\subsection{Our model}

We here consider a generalization of the viscoelastic motor model presented in ref. 55. To start with, we assume that a motor with coordinate $x$ along a microtubule elastically couples to the cargo and exerts the force $f_{\text {ext }}=k_{L}(x-y)$ on it, where the spring constant is $k_{L}$. The motor itself undergoes diffusion, 
most generally characterized by a friction memory $\eta(t)$, on the microtubule. The motor-microtubule binding potential $U(x, \zeta(t))$ reflects the spatial periodicity $L$ of the microtubule. The potential depends on the dynamic motor conformation $\zeta(t)$, the motor's internal degree of freedom. Our model for the coupled equations of motor and cargo coordinates $y$ and $x$ then reads

$$
\begin{gathered}
\int_{-\infty}^{t} \eta_{\mathrm{c}}\left(t-t^{\prime}\right) \dot{y}\left(t^{\prime}\right) \mathrm{d} t^{\prime}=-k_{L}(y-x)+\xi_{\mathrm{c}}(t) \\
\int_{-\infty}^{t} \eta\left(t-t^{\prime}\right) \dot{x}\left(t^{\prime}\right) \mathrm{d} t^{\prime}= \\
k_{L}(y-x)-\frac{\partial}{\partial x} U(x, \zeta(t)) \\
-f_{0}+\xi(t)
\end{gathered}
$$

where the memory kernels $\eta, \eta_{\mathrm{c}}$ and the corresponding autocorrelation functions of the unbiased thermal colored Gaussian noises $\xi$, $\xi_{\mathrm{c}}$ are related by FDTs of the type (2). Furthermore we assume that the noise sources $\xi_{\mathrm{c}}(t)$ and $\xi(t)$ are uncorrelated. The term $f_{0}$ in eqn (3b) is a constant external loading force which can oppose the fluctuation-induced transport and stop it. Such a force can, for instance, be applied in vivo by an optical tweezer or by using magnetic beads. We assume further that the dynamical motor conformations $\zeta(t)$ can take two values $\zeta_{1}$ and $\zeta_{2}$. The binding potential is periodic, $U\left(x+L, \zeta_{1,2}\right)=U\left(x, \zeta_{1,2}\right)$, but spatially asymmetric, as displayed in Fig. 1 in the lower inset. The spatial periodicity guarantees that transport is absent in the absence of conformational fluctuations, which are induced by ATP binding to the molecular motor and subsequent hydrolysis. In terms of statistical mechanics, such transport is forbidden by the symmetry condition of detailed balance at thermal equilibrium in the absence of an energy source. We assume that two consequent conformational switches make one cycle, and thus $U\left(x+L / 2, \zeta_{1}\right)=U\left(x, \zeta_{2}\right)$. Switching between two conformations is considered as symmetric two-state Markovian process with equal transition rates $2 \nu_{\text {turn }}$.

To be more specific, we consider the piecewise linear sawtooth potential with amplitude $U_{0}$ and period $L$. The minimum divides the period in the ratio $p: 1$, and we take the particular value $p=3$. This asymmetry defines the natural direction of transport in direction of positive $x$. The maximal possible load, or stalling force for this potential at zero temperature (i.e., without presence of thermal noise) is easy to deduce, namely, $f_{0}^{\text {stall }}(T=0)=(p+1) U_{0} /(p L)$. In units of thermal energy at room temperature, $k_{\mathrm{B}} T_{\mathrm{r}}=4.1 \times 10^{-21} \mathrm{~J}=4.1 \mathrm{pN}$ $\mathrm{nm}, L=8 \mathrm{~nm}$, we find $f_{0}^{\text {stall }}(T=0) \approx 0.6833 U_{0} /\left(k_{\mathrm{B}} T_{\mathrm{r}}\right) \mathrm{pN}$ for $p=3$. For $T=T_{\mathrm{r}}$ the value for $f_{0}^{\text {stall }}$ will be lower, see below. We choose $U=20 k_{\mathrm{B}} T_{\mathrm{r}}$ in our simulations. This corresponds to $f_{0}^{\text {stall }}(T=0)=$ $13.67 \mathrm{pN}$, about twice the maximal loading force of kinesin II at physiological temperatures. This choice leads to a reasonable stalling force at room temperature.

In this work, we neglect memory effects for the motor particle due to its relatively small size and choose the $\delta$-form $\eta(t)=2 \eta_{\mathrm{m}} \delta(t)$ with regular Stokes friction $\eta_{\mathrm{m}}=6 \pi a_{\mathrm{m}} \zeta_{\mathrm{w}}$, where $a_{\mathrm{m}}$ is the effective radius of the motor molecule and $\zeta_{\mathrm{w}}=1 \mathrm{mPa}$. We take $a_{\mathrm{m}}=100 \mathrm{~nm}$, about 10 times larger than the linear geometrical size of the kinesin molecule in order to account for the enhanced effective viscosity experienced by the motor in the cytosol compared to its value in water. Furthermore, we use the characteristic time scale $\tau_{\mathrm{m}}=L^{2} \eta_{\mathrm{m}} / U_{0}{ }^{*}$ to scale time in the numerical simulations, where $U_{0}^{*}=10 k_{\mathrm{B}} T_{\mathrm{r}}$. For the above parameters, $\tau_{\mathrm{m}} \approx 2.94 \mu \mathrm{s}$. Distance is scaled in units of $L$, and elastic coupling constants in units of $U_{0}^{*} / L^{2} \approx 0.64 \mathrm{pN} \mathrm{nm}^{-1}$. Moreover, we single out the purely viscous component in the memory kernel of cargo as follows: $\eta_{\mathrm{c}}(t)=2 \eta_{\mathrm{c}} \delta(t)+\eta_{\text {mem }}(t)$, where $\eta_{\text {mem }}(t)=\eta_{\alpha} /\left(\Gamma(1-\alpha) t^{\alpha}\right)$.

It should be stressed that even for this particular choice of the memory kernels the studied model cannot be compared directly with the model in ref. 55. Indeed, if we wanted to exclude the dynamics of the cargo variable by projecting the whole dynamics onto the subspace of the motor particle alone, such a reduced dynamics of the motor particle would be described by the Laplace transformed, reduced memory kernel $\tilde{\eta}_{\text {red }}(s)=\eta_{\mathrm{m}}+k_{L} /\left[s+k_{L} /\left(\eta_{\mathrm{c}}+\tilde{\eta}_{\text {mem }}(s)\right)\right]$, where $\tilde{\eta}_{\text {mem }}(s)=\eta_{\alpha} s^{\alpha-1}$ is the Laplace transform of the kernel $\eta_{\text {mem }}(t)$ above. This can be shown for any binding potential $U(x, \zeta(t))$ by formally solving eqn (3a) for the Laplace-transformed variable $y(t)$ using the Laplace-transform method and substituting the result into the Laplace-transformed eqn (3b). Hence, the model of ref. 55 can only be reproduced in the formal limit of an infinitely rigid spring constant with $k_{L} \rightarrow \infty$, upon identifying $\eta_{\mathrm{m}}+\eta_{\mathrm{c}}$ with $\eta_{0}$ in ref. 55, and $\eta_{\text {mem }}(t)$ with $\eta_{\mathrm{m}}(t)$ therein, as well as by adopting the same model of binding potential $U(x, \zeta(t))$. However, we are interested in realistically soft linkers and possible effects they can introduce, especially when a fluctuating binding potential acts on the motor. Indeed, when a normally diffusing particle becomes coupled to an anomalously slow one, who will win and enforce its type of behavior, the normal one, or the anomalous one? Or maybe they will find a compromise? The answer to this question is not trivial. In the absence of the binding potential, one intuitively expects that the anomalously slow diffusing particle will asymptotically enslave the normal particle. However, it is not clear what happens for a fluctuating binding potential. This circle of questions has not been studied before, neither in the theory of anomalous diffusion and transport, nor in the field of molecular motors. To elucidate these points is one of the primary goals in this work. Moreover, in this paper we study a different model of the binding potential, and consider a smaller value of $\alpha=0.4$ in numerical simulations.

When the cargo particle is not coupled to the motor $\left(k_{L} \rightarrow 0\right)$, its free diffusion is described by the $\mathrm{MSD}^{53}$

$$
\left\langle\delta y^{2}(t)\right\rangle=2 D_{\mathrm{c}} t E_{1-\alpha, 2}\left(-\left[t / \tau_{\mathrm{in}}\right]^{1-\alpha}\right),
$$

where $E_{\mathrm{a}, \mathrm{b}}(z)=\sum_{n=0}^{\infty} z^{n} / \Gamma(a n+b)$ is the generalized MittagLeffler function, $D_{\mathrm{c}}=k_{\mathrm{B}} T / \eta_{\mathrm{c}}$ represents the normal diffusion coefficient, and $\tau_{\text {in }}=\left(\eta_{\mathrm{c}} / \eta_{\alpha}\right)^{1 /(1-\alpha)}$ is a time constant separating initially normal diffusion, $\left\langle\delta y^{2}(t)\right\rangle \approx 2 D_{\mathrm{c}} t$ at $t \ll \tau_{\text {in }}$, and subdiffusion, $\left\langle\delta y^{2}(t)\right\rangle \approx 2 D_{\alpha} t^{\alpha} / \Gamma(1+\alpha)$ for $t \gg \tau_{\text {in. }}$. Furthermore, in accordance with the methodology in ref. 30 and 34 we approximate the memory kernel by a sum of $N$ weighted exponentials

$$
\eta_{\mathrm{mem}}(t) \approx \eta_{\mathrm{mem}}\left(t, \nu_{0}, b, N\right)=\sum_{i=1}^{N} k_{i} \exp \left(-\nu_{i} t\right)
$$


with the underlying fractal scaling $\nu_{i}=\nu_{0} / b^{i-1}$ and $k_{i} \propto \nu_{i}^{\alpha}$. This form ensures a power-law scaling, $\eta_{\text {mem }}\left(h t, \nu_{0}, b, N\right)=$ $h^{-\alpha} \eta_{\text {mem }}\left(t, h \nu_{0}, b, N\right)$, for $t$ values well within the time-interval between two short and long time cutoffs $\tau_{\min }=\nu_{0}{ }^{-1}$ and $\tau_{\max }=$ $\tau_{\min } b^{N-1}$. The dilation parameter $b>1$ controls the accuracy of the approximation, which exhibits small logarithmic oscillations with respect to the exact power law, and $\nu_{0}$ corresponds to a highfrequency cutoff reflecting the atomic/molecular nature of any physical condensed environment. In this respect, any physical fractal, either spatial or in time, has minimal and maximal ranges, defined in our case by the ratio $\tau_{\max } / \tau_{\min }=b^{N-1}$, and even a rough decade scaling with $b=10$ allows one to approximate the powerlaw with an accuracy of several percents for $\alpha=0.4$, further increased to a one hundredth of a percent already for $b=2$, see in ref. 52. Similar expansions are well known in the theory of anomalous relaxation. ${ }^{66}$

This Markovian embedding approach provides a foundation for excellent numerical treatment of the GLE dynamics with power-law memory kernel. A convenient parameterization uses

$$
k_{i}=\nu_{0} \eta_{\mathrm{eff}} \frac{b^{1-\alpha}-1}{b^{(i-1) \alpha\left[b^{N(1-\alpha)}-1\right]}},
$$

where $\int_{0}^{\infty} \eta_{\text {mem }}(t) \mathrm{d} t=\eta_{\text {eff }}$ characterizes a largely enhanced macroscopic friction coefficient in the long time limit $t \gg \tau_{\max }$. The number $N$ of auxiliary modes controls the maximal range of the subdiffusive dynamics, which eventually turns normal, $\left\langle\delta y^{2}(t)\right\rangle \sim 2 D_{\text {eff }} t$ for $t \gg \tau_{\max }$ with $D_{\text {eff }}=k_{\mathrm{B}} T /\left(\eta_{\text {eff }}+\eta_{\mathrm{c}}\right)$. Note that the fractional friction coefficient is related to the effective friction by $\eta_{\alpha}=\eta_{\text {eff }} \tau_{\max }^{\alpha-1} / r$. Here, $r=\frac{C_{\alpha}(b)}{\Gamma(1-\alpha)} \frac{b^{1-\alpha}}{b^{1-\alpha}-1}\left[1-b^{-N(1-\alpha)}\right]$ is a numerical coefficient of order of unity, $r \approx 0.93$ for $N \geq 5$ at $\alpha=0.4$ and $b=10$, with $C_{0.4}(b) \approx 1.04$. The effective relative friction coefficient $\tilde{\eta}_{\text {eff }}=\eta_{\text {eff }} / \eta_{\mathrm{c}}$, which is also the effective viscosity of cytosol $\tilde{\zeta}_{\text {eff }}$ relative to water since we assume that the friction coefficient is proportional to the viscosity, is used as an important parameter in our simulations, see below. It defines the time range of subdiffusion, from $\tau_{\text {in }}=\tau_{\max } / \tilde{\eta}_{\text {eff }}^{1 /(1-\alpha)}$ to $\tau_{\max }$. For example, for $\tilde{\eta}_{\text {eff }}=10^{3}$ and $\alpha=0.4$ one expects that subdiffusion will extend over 5 decades in time. According to this important, nontrivial but simple relation, the relative increase of the effective friction controls the relative time range of subdiffusion, $\tilde{\eta}_{\text {eff }}^{1 /(1-\alpha)}$, independently of $b$ and $N$.

The above approximation allows one to replace the nonMarkovian GLE dynamics with a higher dimensional Markovian dynamics by introduction of the $N$ auxiliary Brownian quasiparticles with coordinates $y_{i}$ accounting for internal viscoelastic degrees of freedom. ${ }^{34}$ In the present case,

$$
\begin{aligned}
\eta_{\mathrm{m}} \dot{x} & =f(x, \zeta(t))-k_{L}(x-y)+\sqrt{2 \eta_{\mathrm{m}} k_{\mathrm{B}} T} \xi_{\mathrm{m}}(t), \\
\eta_{\mathrm{c}} \dot{y} & =k_{L}(x-y)-\sum_{i=1}^{N} k_{i}\left(y-y_{i}\right)+\sqrt{2 \eta_{\mathrm{c}} k_{\mathrm{B}} T} \xi_{0}(t), \\
\eta_{i} \dot{y}_{i} & =k_{i}\left(y-y_{i}\right)+\sqrt{2 \eta_{i} k_{\mathrm{B}} T} \xi_{i}(t),
\end{aligned}
$$

where $f(x, \zeta(t))=-\partial U(x, \zeta(t)) / \partial x-f_{0}$, and $\eta_{i}=k_{i} / \nu_{i}$. Furthermore, the $\xi_{i}(t)$ are uncorrelated white Gaussian noise terms of unit intensity, $\left\langle\xi_{i}\left(t^{\prime}\right) \xi_{j}(t)\right\rangle=\delta_{i j} \delta\left(t-t^{\prime}\right)$, which are also uncorrelated with the white Gaussian noise sources $\xi_{0}(t)$ and $\xi_{\mathrm{m}}(t)$. To have a complete equivalence with the stated GLE model in eqn (3a) to (5), the initial positions $y_{i}(0)$ are sampled from a Gaussian distribution centered around $y(0),\left\langle y_{i}(0)\right\rangle=y(0)$ with variances $\left\langle\left[y_{i}(0)-y(0)\right]^{2}\right\rangle=k_{\mathrm{B}} T / k_{i}$. The stochastic variable $\zeta(t)$ is described by a symmetric two-state Markovian process with identical transition rates $\nu=2 \nu_{\text {turn }}$. This corresponds to the simplest model of molecular motors considered as flashing ratchets. ${ }^{2,41,45}$ Numerical solutions of the set of eqn (7) are performed with a time step $\delta t \ll \nu^{-1}$. The variable $\zeta(t)$ alternates its state with probability $\nu \delta t$ at each time step, or continues to stay in the same state with probability $1-\nu \delta t$. This is decided upon comparison of a random number, uniformly distributed between zero and one, with $\nu \delta t$.

\subsection{Thermodynamic efficiency and energetic efficiency of the cargo delivery}

The thermodynamic efficiency of anomalous Brownian motors has been addressed quite recently. ${ }^{52,53}$ It turns out that the general approach developed for normal motors ${ }^{42,67}$ can be almost directly applied. It yields, however, several important new results in the anomalous transport regime. Generically, the work performed by the potential fluctuations induced by the enzyme turnover, or the input energy pumped into directed motion is $E_{\text {in }}(t)=\int_{0}^{t} \frac{\partial}{\partial t} U(x, t) \mathrm{d} t .^{67}$ This can be evaluated within the considered model, as a sum of potential energy jumps $\Delta U\left(x\left(t_{i}\right)\right)$ occurring at random instants of time $t_{i}$ marking the cyclic conformational transitions $1 \rightarrow 2 \rightarrow 1 \rightarrow \cdots$. This input energy is invested in work against the external loading force $f_{0}$. The corresponding useful work is $W_{\mathrm{use}}(t)=f_{0} \delta x(t)$. The rest is used to overcome the dissipative influence of the environment, and thus converted to heat. The thermodynamic efficiency of isothermal motors is thus $R_{\mathrm{th}}(t)=\left\langle W_{\text {use }}(t)\right\rangle /\left\langle E_{\text {in }}(t)\right\rangle$, after averaging over many ensemble realizations. Clearly, $R_{\mathrm{th}}=0$ for $f_{0}=0$. This is also very clear from an energetic point of view: by relocation from one place to another neither the potential energy of the cargo, nor that of the motor has been changed. Such behavior corresponds to the normal modus operandi of motors as kinesin which is very different from other molecular machines such as ion pumps, which are primarily transferring ions against an electrochemical potential gradient, i.e., effecting an increase of the electrochemical potential of the ions. Anomalously slow transport introduces principally new features for $f_{0}>0$. Namely, for anomalous transport the useful work done against $f_{0}$ scales sublinearly with time, $\left\langle W_{\text {use }}(t)\right\rangle \propto$ $f_{0} t^{\alpha_{\text {eff }}}$, while the input energy scales linearly, $\left\langle E_{\text {in }}(t)\right\rangle \propto t$, the latter being proportional to the mean number of potential fluctuations.

By the same token as fractional transport cannot be characterized by a constant mean velocity, it also cannot be characterized by any mean power or useful work in a stationary regime. One way around this is to define a fractional power and 
fractional efficiency. ${ }^{52,53}$ Or, one interprets physically the nonconstant thermodynamic efficiency $R_{\mathrm{th}}$. In the present case it decays algebraically in time, $R_{\mathrm{th}}(t) \propto 1 / t^{1-\alpha_{\text {eff }}}$ and can still be comparatively high even for large times, as shown below. In the anomalous transport regime, asymptotically $R_{\mathrm{th}}(t) \rightarrow 0$ independently of $f_{0}$. Even though the useful work performed against $f_{0}$ is always finite, a benchmark of any genuine Brownian motor, it becomes a negligible portion of the input energy in the course of time. The input energy is spent mostly to overcome the dissipative influence of the environment, that effects a hugely enhanced effective viscosity. It is dissipated as heat, $Q(t)=\left\langle E_{\text {in }}(t)\right\rangle-\left\langle W_{\text {use }}(t)\right\rangle$.

The primary task of molecular motors such as kinesin consists in the delivery of various cargos to certain destinations, and not in the increase of their own potential energy. For this reason, numerous Stokes efficiencies have been defined in addition to $R_{\mathrm{th}}{ }^{68-70}$ Which of them is most appropriate remains somewhat vague, especially with respect to anomalous transport. ${ }^{53}$ We proposed a different measure to quantify the motor performance at $R_{\mathrm{th}}=0$, namely, the energetic delivery performance. ${ }^{55}$ It reflects the optimization of the mean delivery velocity per energy spent. Quantifying the net input energy by the number of enzyme turnovers, a natural definition is $D=d /\left(t\left\langle N_{\text {turn }}\right\rangle\right)$, where $d$ is the delivery distance reached during time $t$, after $\left\langle N_{\text {turn }}\right\rangle$ cyclic turnovers on average. Clearly, $\left\langle N_{\text {turn }}\right\rangle=$ $\nu_{\text {turn }} t$, and for an ideal motor in the tight coupling power stroke regime, whose processive motion is perfectly synchronized with the turnovers of the "catalytic wheel, ${ }^{71} d=L \nu_{\text {turn }} t$, and therefore $D_{\text {ideal }}=L^{2} \nu_{\text {turn }} / d$. For any given $d$, the increase of $\nu_{\text {turn }}$ leads to a linearly increased delivery performance. However, in reality there will always exist deviations from this idealization.

\section{Results and discussion}

In our simulations we use $N=10, b=10$, and $\nu_{0}=100$ for the Markovian embedding, yielding $\tau_{\max } \approx 29.4 \mathrm{~s}$ for $\tau_{\mathrm{m}}=2.94 \mu \mathrm{s}$. The numerical solution of the stochastic differential eqn (7) was achieved via the stochastic Heun method ${ }^{72}$ in CUDA on NVIDIA Kepler graphical processor units. The time step of the integration was $\delta t=5 \times 10^{-3}$ in scaled units, and the terminal time was $10^{6}(2.94 \mathrm{~s})$ in most simulations. We considered a cargo with radius $a=300 \mathrm{~nm}\left(\eta_{\mathrm{c}} / \eta_{\mathrm{m}}=3\right)$ and two different values of $\tilde{\eta}_{\text {eff }}=3 \times 10^{4}$, the 'larger' effective viscosity, and $\tilde{\eta}_{\text {eff }}=$ $3 \times 10^{3}$, the 'smaller' one. This corresponds to two different values of the subdiffusion coefficient $D_{\alpha}^{(1)} \approx 171 \mathrm{~nm}^{2} \mathrm{~s}^{-0.4}$, similar to typical values measured for magnetosomes in ref. 17 and the ten times larger $D_{\alpha}^{(2)} \approx 1710 \mathrm{~nm}^{2} \mathrm{~s}^{-0.4}$, corresponding to a smaller particle. Moreover, two different values for the elastic spring constant were used, $k_{L}^{(1)}=0.32 \mathrm{pN} \mathrm{nm}^{-1}$ ('strong'), which corresponds to measurements in vitro, ${ }^{57}$ and a ten times softer spring $k_{L}^{(2)}=0.032 \mathrm{pN} \mathrm{nm}^{-1}$ ('weak'), in accordance with recent results in ref. 18 in living cells. Finally, $\nu_{\text {turn }}^{(1)}=85 \mathrm{~Hz}$ and $\nu_{\text {turn }}^{(2)}=17 \mathrm{~Hz}$ denote the 'fast' and 'slow' turnover frequencies. According to these parameters we consider the six different parameter sets listed in Table 1.
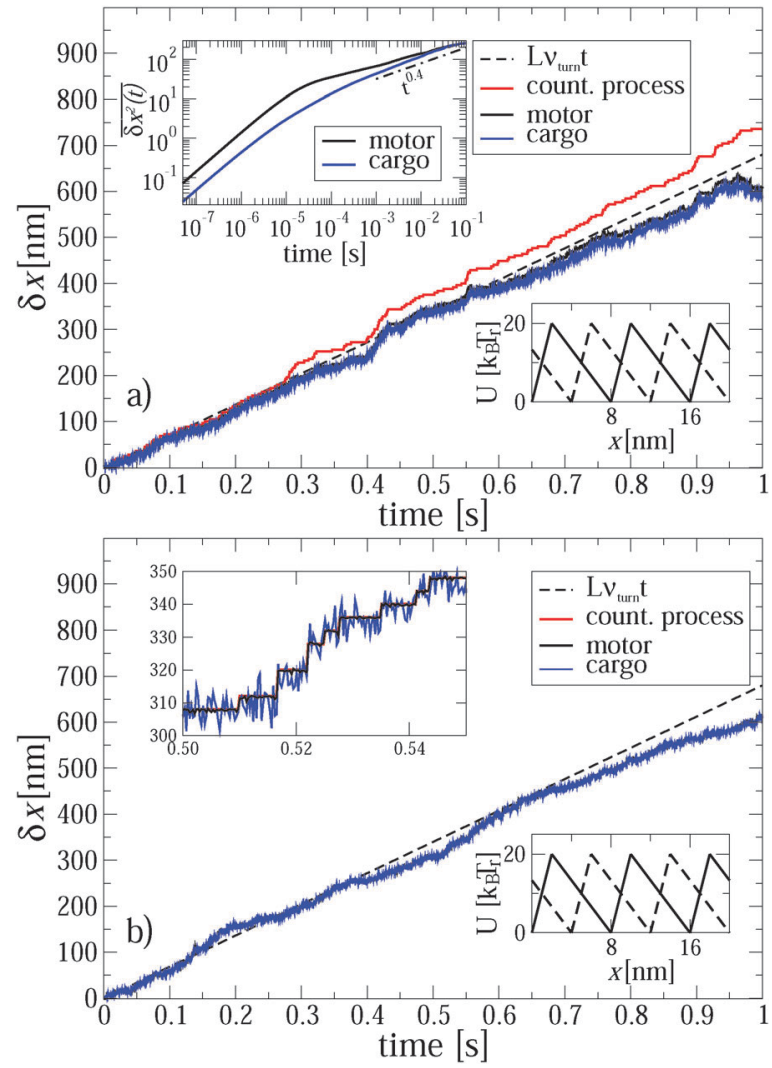

Fig. 1 Positions of the motor (black line) and cargo (blue line) versus time for a single trajectory realization in the case of anomalous transport (part a, set $S_{1}$, see Table 1 for the parameters corresponding to various sets $S_{i}$ ) and normal transport (part $\mathrm{b}$, set $\mathrm{S}_{2}$. Turnover frequency $\nu_{\text {turn }}=85 \mathrm{~Hz}$ ). The realizations of counting process (number of potential switches multiplied with potential half-period $L / 2$ ) are depicted by red lines (difficult to detect in part $b$ because of a perfect synchronization). The broken black lines depict the dependence of the averaged (over many trajectory realizations) position of motor on time in the case of a perfect synchronization (ideal power stroke like mechanism). The upper inset in part a shows diffusion of coupled motor and cargo in the absence of binding potential. The position variances were obtained using a corresponding single-trajectory time averaging, as described in the text. The upper inset in the part $b$ magnifies a part of motor and cargo trajectories making the step-wise motion of motor obvious. It is perfectly synchronized with the potential switches. Cargo randomly fluctuates around the motor position. The lower insets show two conformations of the binding potential.

Table 1 Parameter sets

\begin{tabular}{llll}
\hline Set & $\tilde{\eta}_{\text {eff }}$ & $k_{L} / \mathrm{pN} \mathrm{nm}^{-1}$ & $\nu_{\text {turn }} / \mathrm{Hz}$ \\
\hline $\mathrm{S}_{1}$ & $3 \times 10^{4}$ & 0.320 & 85 \\
$\mathrm{~S}_{2}$ & $3 \times 10^{3}$ & 0.320 & 85 \\
$\mathrm{~S}_{3}$ & $3 \times 10^{3}$ & 0.032 & 85 \\
$\mathrm{~S}_{4}$ & $3 \times 10^{4}$ & 0.032 & 85 \\
$\mathrm{~S}_{5}$ & $3 \times 10^{4}$ & 0.320 & 17 \\
$\mathrm{~S}_{6}$ & $3 \times 10^{4}$ & 0.032 & 17
\end{tabular}

In Fig. 1a we illustrate both the active and passive motion of the cargo and motor particles for single trajectories. Consider first the case that the motor particle is detached from the microtubule. If motor and cargo are unconnected, the motor particle according to our model assumption would diffuse 
normally at all times, $\left\langle(\delta \mathbf{r})^{2}\right\rangle \propto D_{1} t$, while the larger cargo particle would exhibit anomalous diffusion, $\left\langle(\delta \mathbf{r})^{2}\right\rangle \propto D_{\alpha} t^{\alpha}$, over a large intermediate time range. Initially, both particles diffuse normally as the viscoelastic friction is not relevant at such early times. Interestingly, a similar feature, i.e., initially normal diffusion without any persistence or anti-persistence in the motion was found experimentally for lipid droplets in ref. 65 . When both motor and cargo particles are harmonically coupled, the motor particle initially diffuses normally but eventually becomes enslaved by the cargo particle, and both subdiffuse. This is shown in the top inset of Fig. 1a, where we plot the single trajectory MSD in terms of the sliding average

$$
\overline{\delta x^{2}(t)}=\frac{1}{\mathscr{T}-t} \int_{0}^{\mathscr{T}-t} \delta x^{2}\left(t \mid t^{\prime}\right) \mathrm{d} t^{\prime}
$$

over the particle position variance $\delta x^{2}\left(t \mid t^{\prime}\right)=\left[x\left(t+t^{\prime}\right)-x\left(t^{\prime}\right)\right]^{2}$. In the limit $t \ll \mathscr{T}$ this quantity provides the same information as the ensemble average $\left\langle\delta x^{2}(t)\right\rangle$, since the considered viscoelastic diffusion is ergodic. ${ }^{30,73,74}$ We note that, on average, no mean displacement $\overline{\delta x(t)}$ of the motor-cargo complex occurs. This is in accordance with our intuition: the subdiffusing particle eventually enslaves the normal one, in the absence of external forces acting on the latter.

However, once the motor is attached to the microtubular track, it processively steps in the direction defined by the asymmetry of the ratchet potential, and now the cargo has to move along with the motor. This is seen in the main part of Fig. 1a. Initially, the motor steps are perfectly coupled to the potential fluctuations, where it should be noted that a particular realization of the counting process in units of $L / 2$ in this figure is faster than the average value corresponding to the optimal transport distance $d=L \nu_{\text {turn }}^{(1)} t, c f$. the broken line. Indeed, different realizations of single enzyme dynamics yield different realizations of the potential fluctuations and the above optimal transport distance is the ensemble average over many trajectories. The particular realization can be both faster and slower, with a typical fluctuation in the number of steps of the order of $\sqrt{\nu_{\text {turn }} t}$. After some transient time one can clearly see the motor backstepping. Moreover, the cargo particle always fluctuates around the motor position, but on average it lags somewhat behind the motor. It is not obvious from this figure that the transport is anomalously slow and not just corresponds to some suboptimal mean motor velocity $v<$ $v_{\text {opt }}=L \nu_{\text {turn }}$. However, the transport is indeed anomalous, $\langle\delta x(t)\rangle \propto t^{\alpha_{\text {eff }}}$, after an initially normal transport regime as can be deduced after averaging over 1000 different trajectory realizations, as shown in Fig. 2 . This allows us to extract the anomalous transport exponent $\alpha_{\text {eff. }}$ As already verified in our previous studies of viscoelastic subdiffusive dynamics in periodic nonlinear potentials, the value of this $\alpha_{\text {eff }}$ is generally timedependent. However, it relaxes to a long-time limiting value in the course of time, which is displayed in Fig. 3 as function of the load $f_{0}$ for different sets of parameters.

It is very important for physical reasons that the cargo lags behind the motor, following at a certain mean distance with
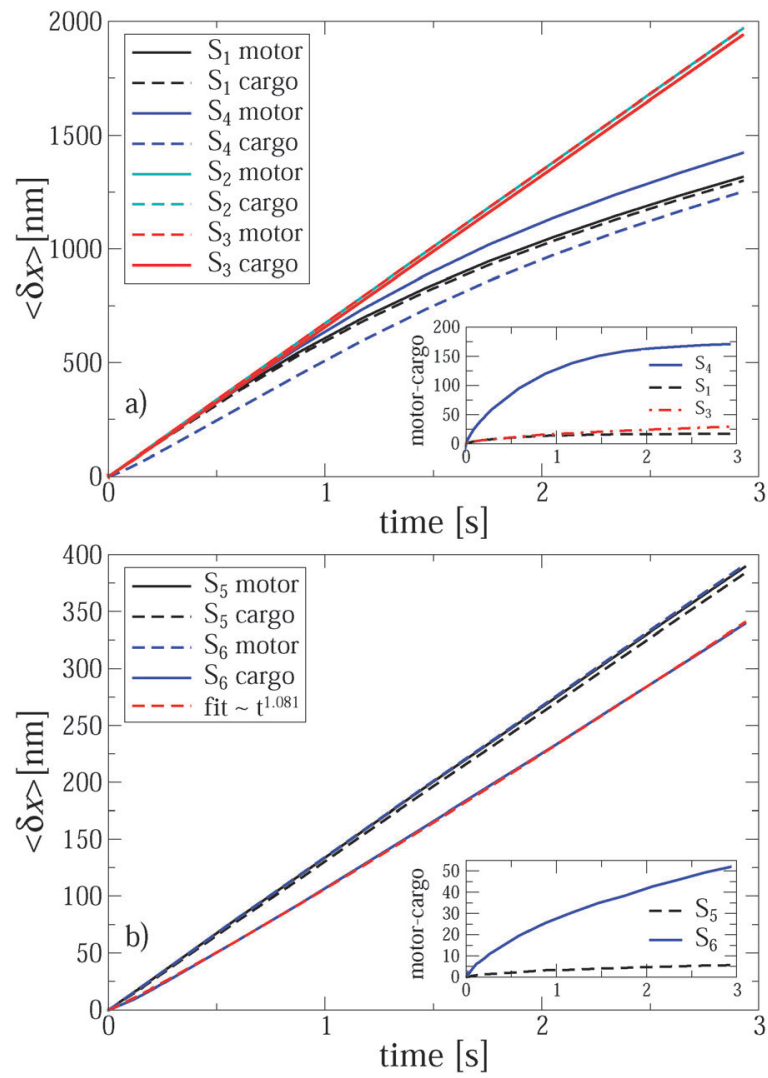

Fig. 2 Ensemble-averaged trajectories $\left(10^{3}\right.$ different realizations) of motor and cargo particles for different sets of parameters at two values of turnover frequencies, $85 \mathrm{~Hz}(\mathrm{a})$, and $17 \mathrm{~Hz}$ (b). The opposing force is absent, $f_{0}=0$, in all cases. Insets show the differences between the motor and cargo positions for the chosen sets. Transport of smaller particles is normal. (a) Within the anomalous transport regime of larger particles, the motor-cargo distance increases and saturates at about $17 \mathrm{~nm}$ for $\mathrm{S}_{1}$ and about $170 \mathrm{~nm}$ for $\mathrm{S}_{4}$. These values largely exceed the corresponding thermal rms distances of $3.58 \mathrm{~nm}$ and $11.32 \mathrm{~nm}$, respectively. This means that weaker linker can be unfolded during the transport of larger particle, or even break down. (b) For a smaller turnover frequency, anomalous transport becomes normal both for motor and cargo in the case of strong linker, $S_{5}$. Surprisingly, for a weak linker, $S_{6}$, the motor operates normally, while cargo enters a paradoxical super-transport regime with exponent $\alpha_{\text {eff }}$ $\approx 1.081$, even if it moves slower than motor.

fluctuations around it. Therefore, it makes sense to consider two effective exponents $\alpha_{\text {eff }}$ for the motor and cargo separately. For a stronger linker, both exponents are very similar because the average distance between the motor and the cargo is relatively small, see the inset in Fig. 2a. Even in the strongly anomalous case $S_{1}$, the average distance saturates at around $\delta x=17 \mathrm{~nm}$, which largely exceeds the corresponding amplitude of the thermal fluctuations, given by the root mean square (rms) $\delta x_{\mathrm{T}}=\sqrt{k_{\mathrm{B}} T_{\mathrm{r}} / k_{L}} \sim 3.58 \mathrm{~nm}$. The elastic spring energy corresponding to the above average distance is $E_{L}=k_{L}^{(1)}(\delta x)^{2} / 2 \sim$ $46.24 \mathrm{pN} \mathrm{nm} \sim 11 k_{\mathrm{B}} T_{\mathrm{r}}$. It can be easily sustained by a covalent bond. However, for a weaker linker the scenario becomes highly nontrivial and in fact strongly depends on the character of the transport regime. In this case, the difference between two exponents $\alpha_{\text {eff }}$ for motor and cargo can be essential, see in 


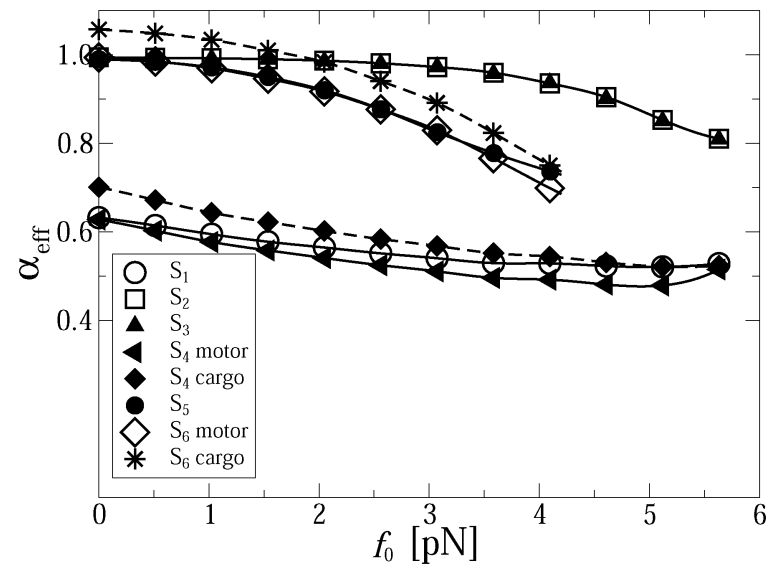

Fig. 3 Effective transport exponents $\alpha_{\text {eff }}$ of the motor and cargo as functions of loading force $f_{0}$ for various sets of parameters, as deduced from the ensemble-average at the end point of simulations (2.94 s). These two values of $\alpha_{\text {eff }}$ differ significantly for weak linker and large cargo, the cases $\mathrm{S}_{4}$ and $\mathrm{S}_{6}$ shown in this plot. For other cases, the differences are much less pronounced. For this reason, only $\alpha_{\text {eff }}$ for the motor is displayed for the other sets. Notice that $\alpha_{\text {eff }}$ of the cargo can be larger than one for a normally operating motor in the case of a weak linker, $\mathrm{S}_{6}$.

Fig. 3 for a larger cargo. For a smaller cargo in the normal transport case $S_{3}$, the distance is about $29 \mathrm{~nm}$ at the end of the simulation, see the inset in Fig. 2a, and the spring energy is about $3.3 k_{\mathrm{B}} T_{\mathrm{r}}$ only. However, in the strongly anomalous regime $\mathrm{S}_{4}$, the mean distance increases to $170 \mathrm{~nm}$ and the spring energy rises accordingly until about $111 k_{\mathrm{B}} T_{\mathrm{r}}$. This is already a typical energy of a covalent bond. Therefore, such a linker, considered as globular entropic spring, will be definitely unfolded to reach its maximal length, which can indeed be around $150 \mathrm{~nm}$ when it is fully stretched and is sufficiently long. ${ }^{75}$ However, the most probable scenario is that such a linker will simply break or, alternatively, the cargo will dissociate from the linker and will be lost by the motor. This is the first important result of this work. Namely, to transfer a large cargo in a strongly anomalous transport regime the linker must be sufficiently strong. With these caveats in mind, we consider the case $\mathrm{S}_{4}$ in more detail, as it also reveals another remarkable feature, which is expected to be present for stronger linkers, as well, albeit to a lesser degree. Namely, in the case $S_{4}$ the motor moves faster than in the case of a stronger linker for $S_{1}$, see Fig. 2a. This is a remarkable observation, which was not expected a priori. Given this feature, the motor can be expected to perform a larger work against an external load $f_{0}$ for a weaker linker. Moreover, on decreasing the turnover frequency down to $17 \mathrm{~Hz}$ (see the case $\mathrm{S}_{6}$ in Fig. 2b) the motor operates normally, with the same mean velocity as in case $S_{5}$ corresponding to the optimal motor regime at this frequency. However, the cargo paradoxically enters a super-transport regime with a scaling exponent $\alpha_{\text {eff }} \approx 1.081$, see also Fig. 3, while moving slower than the motor. This means that the transport exponent $\beta=$ $2 \alpha_{\text {eff }}$ can, in principle, become larger than two, i.e. the observed motor-mediated diffusion of the cargo can be transiently superballistic. This is our second nontrivial and surprising result.
The explanation of this seemingly paradoxical behavior is quite simple. Namely, it occurs because the distance between the motor and cargo increases sublinearly in time, before entering the saturation regime. To realize this, let us assume first that this mutual distance grows linearly in time. Then, the cargo distance would also grow linearly with an effective velocity equal to the motor velocity minus the velocity of the mutual distance growth. However, if the mutual distance increases only sublinearly, i.e. with a velocity which drops in time, see inset in Fig. 2b, it appears that the cargo moves faster than in the normal transport regime characterized by the effective velocity. Paradoxically, an ever increasing delay of the cargo motion past the optimally operating motor due to the weakness of the motor-cargo linker can be interpreted as a super-transport of cargo. However, upon a saturation of the motor-cargo distance this transient regime will be over. This example shows that it can be very misleading to associate the value of anomalous transport exponent $\alpha_{\text {eff }}$ with the speed of transport.

For $\mathrm{S}_{1}$ and $f_{0}=0$ shown in Fig. $1 \mathrm{a}, \alpha_{\mathrm{eff}} \approx 0.63$, both for the motor and cargo. The same value also characterizes the motor in the case $\mathrm{S}_{4}$. However, for the cargo the transport exponent here is larger, $\alpha_{\text {eff }} \approx 0.70$, even if it moves absolutely slower, an interesting feature by itself. This once again indicates that there is no simple general relation between the value of the transport exponent and the transport speed, contrary to common belief. The observed features explain the origin of the superdiffusive exponent in the range $\beta=1.2-1.4$, in spite of the low value of the free subdiffusion exponent $\alpha=0.4$ in ref. 17 . However, for a smaller particle (or rather for a smaller $\eta_{\alpha}$ in our model) corresponding to the case $\mathrm{S}_{2}$, the transport becomes normal at the same flashing frequency and without biasing back load, $f_{0}=0$, as demonstrated in Fig. 1b. Interestingly, the corresponding single trajectory realization in Fig. 1b does not correspond to a larger transport distance at $t=1 \mathrm{~s}$ as compared with the anomalous transport in Fig. 1a. This is simply because the number of turnovers performed until this time is larger in Fig. 1a than in Fig. 1b, for the particular realizations presented. This reflects statistical variations of the corresponding counting process, or, in physical terms, the stochastic nature of single motor proteins. The upper inset in Fig. 1b reveals a perfect synchronization between the potential switches and the motor steps. It renders the red line corresponding to the potential switches barely distinguishable in this plot. One can also see in this upper inset that the cargo particle fluctuates symmetrically around the motor. It does not lag behind the motor on average, in contrast to Fig. 1a. Furthermore, within a transport regime closer to normal transport a much softer linker does not significantly change the results, as demonstrated by Fig. 3 for a smaller cargo. For a larger cargo, though, anomalous transport becomes clearly affected in a very nontrivial way, as discussed above.

Notably, the motor can operate normally if the turnover frequency is reduced, see the results for $\mathrm{S}_{5}$ and $\mathrm{S}_{6}$ at $f_{0}=0$ in Fig. 3. However, the transport of cargo can become both normal (strong linker, $\mathrm{S}_{5}$ ), or even, seemingly paradoxical, anomalously fast, if to judge from the transport exponent $\alpha_{\text {eff }}$ for a weak 


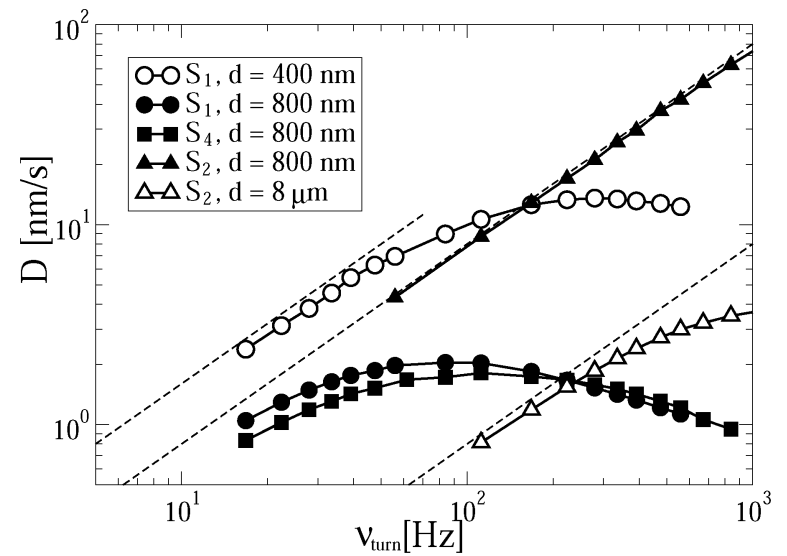

Fig. 4 Cargo transport delivery efficiency $D$ as function of turnover frequency $\nu_{\text {turn }}$ for different sets of parameters and different delivery distances $d$. Broken lines correspond to the ideal power-stroke dependence $D_{\text {ideal }}=L^{2} \nu_{\text {turn }} / d$, for comparison.

linker, $\mathrm{S}_{6}$. This super-transport is in fact absolutely slower than the normal one, see Fig. $2 \mathrm{~b}$ and the related discussion above. These results show that motors can realize both normal and anomalous transport in the viscoelastic cytosol of living cells, where large submicron cargos subdiffuse on the time scale from milliseconds to tens of seconds. Which transport regime will be realized depends specifically on the particle size (or fractional friction coefficient $\eta_{\alpha}$ ), the enzyme turnover frequency $\nu_{\text {turn }}$, and on the linker rigidity. This presents another important result of our work.

Furthermore, if a counter-force $f_{0}>0$ is applied, the anomalous transport regime becomes promoted, as shown in Fig. 3. This effect is primarily due to the reduction of the binding potential amplitude $U_{0}$. Anomalous transport will also emerge immediately for the studied parameters if $U_{0}$ is decreased, for instance, to the lower value $10 k_{\mathrm{B}} T_{\mathrm{r}}$.

The transport efficiency of cargo delivery in the viscoelastic cytosol can be of the same order as that for the motion in a dilute solution, despite the passive subdiffusion. This efficiency is demonstrated in Fig. 4 for realistic turnover frequencies (lower than $200 \mathrm{~Hz}$ ). It persists even for large distances of some $8 \mu \mathrm{m}$. The discussed power stroke mechanism can thus perfectly overcome the subdiffusive resistance of the medium, if the cargo size is not too large. In the anomalous transport regime with strongly decreased transport efficiency there still exists an optimal turnover frequency, which depends on the delivery distance. Indeed, in the anomalous transport regime with increasing turnover frequency or load even normally operating motors do not have sufficient time to relax down to the potential minimum after each potential jump, or escape events become important. This results in backsteps, and after reaching a maximum versus $\nu_{\text {turn }}$ the delivery efficiency will necessarily drop. In the anomalous transport regime, the optimal $\nu_{\text {turn }}^{\text {opt }}(d)$ necessarily decays with increasing delivery distance $d$. Energetically, it makes then less sense to hydrolyze more ATP molecules for efficient cargo delivery. A corresponding optimization can be important for the cell economy.

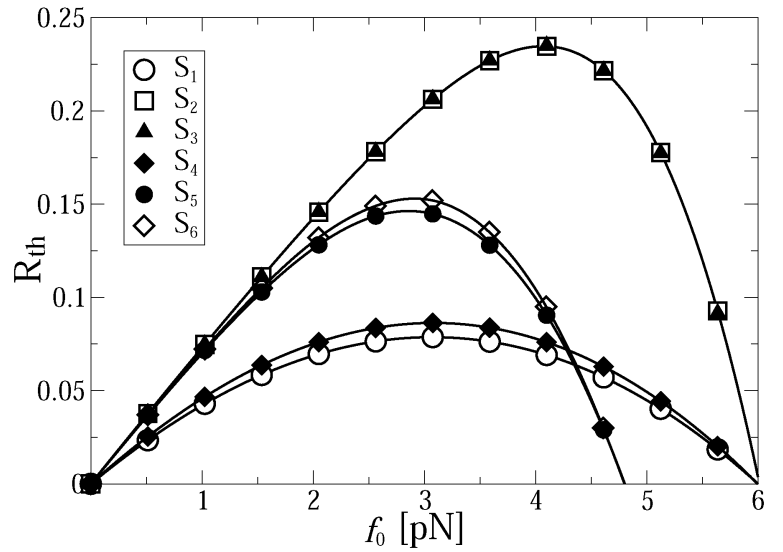

Fig. 5 Motor thermodynamic efficiency as function of loading force $f_{0}$ at the end of simulations (corresponding to $t_{\max }=2.94 \mathrm{~s}$ ) for different sets.

As expected, anomalous transport on a stronger linker is more efficient for realistic turnover frequencies, a fact that agrees with intuition. This is because the cargo follows at a larger distance from the motor in case of a weaker linker.

The thermodynamic efficiency becomes strongly affected by the fact that an increase of $f_{0}$ promotes the onset of the anomalous transport regime, as observed in Fig. 3. Even if the transport was normal for $f_{0}=0$ it may become anomalous under a sufficiently strong external load $f_{0}$. Hence, whenever the transport is anomalous, the thermodynamic efficiency starts to depend on time and vanishes asymptotically. However, it can be quite large even at long times such as $t_{\max } \sim 3 \mathrm{~s}$, see Fig. 5 , where it still amounts to some $23 \%$ at the maximum, see the results for cases $S_{2}$ and $S_{3}$, where the thermodynamic efficiency practically does not depend on the linker rigidity. This is an important result of the present work, which is a priori counterintuitive. Namely, in a highly efficient transport regime, which is closer to normal transport, neither transport exponent $\alpha_{\text {eff }}$ (Fig. 3) nor the thermodynamic efficiency are practically affected by the rigidity of the linker. However, in the low efficiency transport regime, the influence of the linker rigidity becomes evident in Fig. 3 and 5. Remarkably, the thermodynamic efficiency of the motor is slightly larger for a weaker linker. This interesting feature is due to the fact that the motor covers larger distances in this case, as shown in Fig. 2. Interestingly, the stalling force does not depend on $k_{L}$ at all for the studied parameters. The dependence of $R_{\mathrm{th}}\left(t, f_{0}\right)$ on the load $f_{0}$ is very illustrative: $R_{\mathrm{th}}\left(t, f_{0}\right)$ vanishes not only at $f_{0}=0$, but also at some $f_{0}^{\text {stall }}\left(T, U_{0}, \nu_{\text {turn }}\right)$. This defines the stalling force, which is time-independent but strongly depends on potential amplitude, temperature, and driving frequency. The thermodynamic efficiency has thus a maximum at some optimal loading force $f_{0}^{\text {opt }}(t)$, and $R_{\mathrm{th}}\left(t, f_{0}^{\text {opt }}\right)$ can be relatively high in the anomalous transport regime, for a sufficiently high potential amplitude $U_{0}$, comparable with the maximal thermodynamic efficiency of kinesins in the normal regime of about $50 \%$. The dependence of $R_{\mathrm{th}}\left(t, f_{0}\right)$ on $f_{0}$ is strongly asymmetric in the thermodynamically highly efficient regime. However, it becomes more symmetric in a low efficient anomalous regime, where it is 


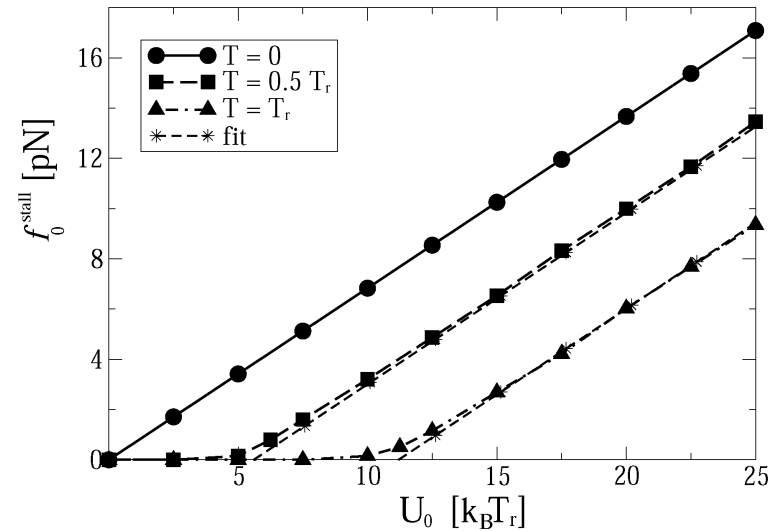

Fig. 6 Stalling force as function of barrier height $U_{0}$ at different temperatures and fixed $\nu_{\text {turn }}=85 \mathrm{~Hz}$. Numerical results are compared with an analytical fit by eqn (9) for $U_{0}>U_{m} T / T_{r}$.

described approximately by the parabolic dependence $R_{\text {th }} \propto$ $f_{0}\left(1-f_{0} / f_{0}^{\text {stall }}\right) / f_{0}^{\text {stall }}$ with $f_{0}^{\text {opt }}=f_{0}^{\text {stall }} / 2$, and a proportionality coefficient which slowly drops in time. This phenomenon of a time-dependence of $R_{\mathrm{th}}(t)$ can be verbalized as fatigue of molecular motors caused by the viscoelasticity of the cytosol. It can be used as direct means to probe our model experimentally.

Indeed, for low-efficient anomalous transport, the dependence of $R_{\text {th }}$ on the load in Fig. 5 is approximately parabolic, with a maximum at $f_{0}^{\text {opt }}=f_{0}^{\text {stall }} / 2$. Counterintuitively, it is slightly larger for a softer linker. This is because the useful work was defined as the work performed by motors against $f_{0}$, and not by the cargo, and that the motors step ahead of their cargos on slightly larger distances for a softer linker. The maximal loading, or stalling force $f_{0}^{\text {stall }}$ is time-independent. It also does not depend on the cargo size. However, it strongly depends on the flashing frequency (Fig. 5), as well as on the potential amplitude and temperature (Fig. 6). Numerical results show that for $U_{0}>U_{\mathrm{m}}\left(\nu_{\text {turn }}\right) T / T_{\mathrm{r}}$,

$$
f_{0}^{\text {stall }}\left(T, U_{0}, \nu_{\text {turn }}\right) \approx \frac{4}{3 L}\left(U_{0}-U_{\mathrm{m}}\left(\nu_{\text {turn }}\right) \frac{T}{T_{\mathrm{r}}}\right),
$$

and a fit to numerical data yields $U_{\mathrm{m}} \approx 11.2 k_{\mathrm{B}} T_{\mathrm{r}}$ at $\nu_{\text {turn }}=$ $85 \mathrm{~Hz}$. The corresponding stalling force becomes $f_{0}^{\text {stall }} \approx 6 \mathrm{pN}$ for $U_{0}=20 k_{\mathrm{B}} T_{\mathrm{r}}$, but is smaller for $\nu_{\text {turn }}=17 \mathrm{~Hz}$ (Fig. 5). From this we conclude that a reasonably strong motor requires binding potential amplitudes larger than some ten $k_{\mathrm{B}} T$. We may interpret the result in eqn (9) in terms of an effective free energy barrier $F_{0}(T)=U_{0}-T S_{0}$ with an effective 'entropy' $S=U_{\mathrm{m}}\left(\nu_{\text {turn }}\right) / T_{\mathrm{r}}$. Then, $f_{0}^{\text {stall }}\left(T, U_{0}, \nu_{\text {turn }}\right) \approx 4 F_{0}(T) / 3 L$ for positive $F_{0}$.

\section{Conclusions}

In this work, we presented a model for active transport of submicron cargo particles by molecular motors in the viscoelastic cytosol of living cells. This model goes significantly beyond our previous discussion in ref. 55 by the inclusion of a realistic elastic linker between the motor and its cargo, giving rise to a number of surprising effects. When decoupled from the motor, the cargo particles subdiffuse and the motor operates normally. As shown here, the effective transport of the coupled motor-cargo complex may be both normal and anomalous, depending on the physical parameters of the system.

The viscoelasticity of the cellular cytosol is taken into account by the formulation of the cargo dynamics in terms of a Generalized Langevin Equation with a memory kernel, which scales in accordance with a power law between two cutoffs. Passive subdiffusion according to our model occurs on time scales between $\tau_{\min }=\tau_{\max } / \zeta_{\text {rel }}^{1 /(1-\alpha)}$ and $\tau_{\max }$, where $\zeta_{\text {rel }}$ is the effective cytosol viscosity relative to the water viscosity. It is distinctly enhanced, $\zeta_{\text {rel }} \gg 1$, for submicron particles. Asymptotically, normal diffusion of the free particle is restored, but with this increased value of $\zeta_{\text {rel }}$. For $\alpha=0.4$ and typical values $\zeta_{\text {rel }} \sim 10^{4}$ or $\zeta_{\text {rel }} \sim 10^{3}$, subdiffusion then persists over about 6.5 or 5 decades in time, respectively. Such transient subdiffusion is described by the multidimensional Markovian embedding with a well-controlled accuracy of the approximation. The molecular motor is described by a variant of the standard Markovian model of flashing Brownian motors with a periodic saw-tooth potential, that randomly fluctuates between two realizations differing by phase, such that two potential flashes correspond to one complete enzyme cycle, that can advance the motor by one spatial period. This model describes, in particular, a perfect power stroke ratchet transport in the case of highly processive molecular motors. It explains how a perfect ratchet mechanism can win over subdiffusional restrictions in living cells. A perfect transport regime with maximal transport efficiency emerges when the motion of the motor is locked onto the potential fluctuations effected by the internal motor turnover. As an important new result, we clearly showed that in order to operate efficiently with a non-vanishingly small stalling force in the viscoelastic environment of living cells the amplitude of the binding potential $U_{0}$ should be no less than around ten $k_{\mathrm{B}} T_{\mathrm{r}}$, or $0.25 \mathrm{eV}$. For a realistic value $U_{0} \sim 20 k_{\mathrm{B}} T_{\mathrm{r}} \sim$ $0.5 \mathrm{eV}$, with a stalling force about 5-6 pN within the present model, depending on the motor operating frequency it turns out that variation of the spring constant of the linker in the experimentally relevant range $k_{L} \sim 0.03-0.3 \mathrm{pN} \mathrm{nm}^{-1}$ does not change significantly the major results in the efficient transport regime for sufficiently small cargos. However, in a strongly anomalous regime emerging for large cargos the weakness of the linker can distinctly influence the transport. In fact, our results show that a weak linker with $k_{L} \sim 0.03 \mathrm{pN} \mathrm{nm}^{-1}$ might simply not be able to sustain anomalous transport of sufficiently large cargos with $a=300 \mathrm{~nm}$ in such a regime. It will be strongly stretched and eventually break, or the cargo will dissociate from it and become lost (other scenarios are also possible). However, if the motor operates slowly and enters the optimal normal regime, such a weak linker may indeed support transport of such large cargos on a smaller spatial scale with cargo entering a paradoxical regime of transient supertransport with $\alpha_{\text {eff }}$ larger than one, the origin of which is rationalized within our model. Furthermore, the cargo delivery efficiency is generally larger for a stronger linker, but the thermodynamic efficiency of the motor is somewhat smaller. 
In our view, these conclusions are interesting and important, and will prompt further studies in this direction.

The crucial point which our model explains is how one and the same motor in the same cell can realize both normal and anomalous transport. The occurrence of a particular transport regime depends on the binding potential amplitude, the fractional frictional strength $\eta_{\alpha}$ of the cargo particle (depending on its size), the external loading force $f_{0}$, and the enzyme turnover frequency. The effective transport exponent $\alpha_{\text {eff }}$ can vary from $\alpha$ to one, and this can easily explain the emergence of anomalously fast diffusion with $\beta=2 \alpha_{\text {eff }}$ mediated by motors in the cells with $\alpha \leq 0.5$. Strikingly enough, the transport can be perfectly normal (in agreement with many experiments), reflecting an almost perfect synchronization between the enzymatic turnovers and the motor's stepping along microtubule. This can be rationalized within a power-stroke mechanism and explains how a power-stroke like operation of molecular motors can win over passive subdiffusion. Conversely, in the presence of a large cargo or otherwise unfavorable parameters such as a high turnover frequency, the viscoelasticity of the environment may enforce a subdiffusive, anomalous transport by the molecular motor. Then, the average distance traveled by the motorcargo compound grows slower than linearly in time.

As a final conclusion, we reaffirmed in a more realistic setting the major qualitative results obtained in ref. 55 based on a more simplistic approach. Thus, the occurrence of anomalous versus normal transport depending on the cargo size and the motor turnover frequencies now appears to be well rationalized. In particular, in order to beat subdiffusion with a power stroke one needs an appreciably strong linker between the motor and its cargo.

We hope that these new results will inspire new experiments, as they should be present in more complex models of molecular motors operating in viscoelastic cytosol. We hope that this can be done in a nearest future.

\section{Acknowledgements}

Support of this research by the German Research Foundation, Grant GO 2052/1-2, as well as funding from the Academy of Finland (FiDiPro scheme) are gratefully acknowledged.

\section{References}

1 R. Kubo, Rep. Prog. Phys., 1966, 29, 255.

2 P. Nelson, Biological Physics: Energy, Information, Life, Freeman and Co., New York, 2004.

3 K. Luby-Phelps, Mol. Biol. Cell, 2013, 24, 2593.

4 T. Odijk, Biophys. J., 2000, 79, 2314.

5 L. Masaro and X. X. Zhu, Prog. Polym. Sci., 1999, 24, 731.

6 R. Holyst, et al., Phys. Chem. Chem. Phys., 2009, 11, 9025.

7 I. Goychuk, Phys. Rev. E: Stat., Nonlinear, Soft Matter Phys., 2012, 86, 021113.

8 G. Guigas, C. Kalla and M. Weiss, Biophys. J., 2007, 93, 316. 9 F. Amblard, et al., Phys. Rev. Lett., 1996, 77, 4470.
10 M. J. Saxton and K. Jacobson, Annu. Rev. Biophys. Biomol. Struct., 1997, 26, 373.

11 H. Qian, Biophys. J., 2000, 79, 137.

12 S. Yamada, D. Wirtz and S. C. Kuo, Biophys. J., 2000, 78, 1736.

13 A. Caspi, R. Granek and M. Elbaum, Phys. Rev. E: Stat., Nonlinear, Soft Matter Phys., 2002, 66, 011916.

14 I. M. Tolić-Nørrelykke, E.-L. Munteanu, G. Thon, L. Oddershede and K. Berg-Sørensen, Phys. Rev. Lett., 2004, 93, 078102.

15 I. Golding and E. C. Cox, Phys. Rev. Lett., 2006, 96, 098102. 16 J. Szymanski and M. Weiss, Phys. Rev. Lett., 2009, 103, 038102. 17 D. Robert, Th.-H. Nguyen, F. Gallet and C. Wilhelm, PLoS One, 2010, 4, e10046.

18 L. Bruno, M. Salierno, D. E. Wetzler, M. A. Desposito and V. Levi, PLoS One, 2011, 6, e18332.

19 J. H. Jeon, V. Tejedor, S. Burov, E. Barkai, C. Selhuber-Unkel, K. Berg-Sørensen, L. Oddershede and R. Metzler, Phys. Rev. Lett., 2011, 106, 048103.

20 J. H. Jeon, M. Leijnse, L. B. Oddershede and R. Metzler, New J. Phys., 2013, 15, 045011.

21 E. Barkai, Y. Garini and R. Metzler, Phys. Today, 2012, 65(8), 29.

22 S. M. A. Tabei, S. Burov, H. Y. Kim, A. Kuznetsov, T. Huynh, J. Jureller, L. H. Philipson, A. R. Dinner and N. F. Scherer, Proc. Natl. Acad. Sci. U. S. A., 2013, 110, 4911.

23 F. Höfling and T. Franosch, Rep. Prog. Phys., 2013, 76, 046602.

24 M. A. Taylor, J. Janousek, V. Daria, J. Knittel, B. Hage, H.-A. Bachor and W. P. Bowen, Nat. Photonics, 2013, 7, 229.

25 S. C. Weber, A. J. Spakowitz and J. A. Theriot, Phys. Rev. Lett., 2010, 104, 238102.

26 M. Weiss, Phys. Rev. E: Stat., Nonlinear, Soft Matter Phys., 2013, 88, 010101(R).

27 M. Magdziarz, A. Weron, K. Burnecki and J. Klafter, Phys. Rev. Lett., 2013, 103, 180602.

28 A. N. Kolmogorov, Dokl. Akad. Nauk SSSR, 1940, 26, 115 (in Russian), English transl. in Selected Works of A. N. Kolmogorov, vol. I, Mechanics and Mathematics, ed. V. M. Tikhomirov, Kluwer, Dordrecht, 1991, pp. 303-307.

29 B. B. Mandelbrot and J. W. van Ness, SIAM Rev., 1968, 10, 422 .

30 I. Goychuk, Phys. Rev. E: Stat., Nonlinear, Soft Matter Phys., 2009, 80, 046125.

31 K. G. Wang and M. Tokuyama, Physica A, 1999, 265, 341.

32 I. Goychuk and P. Hänggi, Phys. Rev. Lett., 2007, 99, 200601.

33 R. Zwanzig, J. Stat. Phys., 1973, 9, 215.

34 I. Goychuk, Adv. Chem. Phys., 2012, 150, 187.

35 R. Zwanzig, Nonequilibrium statistical mechanics, Oxford University Press, Oxford, 2001.

36 R. Kubo, M. Toda and M. Hashitsume, Nonequilibrium statistical mechanics, Springer, Berlin, 2nd edn, 1991.

37 R. J. Ellis and A. P. Minton, Nature, 2003, 425, 27.

38 S. R. McGuffee and A. H. Elcock, PLoS Comput. Biol., 2010, 6, e1000694.

39 N. Hirokawa and R. Takemura, Nat. Rev. Neurosci., 2005, $6,201$. 
40 A. T. Jones, M. Gumbleton and R. Duncan, Adv. Drug Delivery Rev., 2003, 55, 1353.

41 J.-F. Chauwin, A. Ajdari and J. Prost, Europhys. Lett., 1994, 27, 421.

42 F. Jülicher, A. Ajdari and J. Prost, Rev. Mod. Phys., 1997, 69, 1269.

43 F. Jülicher, in Transport and Structure: Their Competitive Roles in Biophysics and Chemistry, Lect. Not. Phys., ed. S. C. Müller, J. Parisi and W. Zimmermann, Springer, Berlin, 1999, vol. 532, p. 46.

44 R. D. Astumian, Science, 1997, 276, 917.

45 R. D. Astumian and M. Bier, Biophys. J., 1996, 70, 637.

46 M. E. Fisher and A. B. Kolomeisky, Proc. Natl. Acad. Sci. U. S. A., 2001, 98, 7748.

47 R. Perez-Carrasco and J. M. Sancho, Biophys. J., 2010, 98, 2591.

48 P. Reimann, Phys. Rep., 2002, 361, 57.

49 I. Goychuk, Chem. Phys., 2010, 375, 450.

50 I. Goychuk and V. Kharchenko, Phys. Rev. E: Stat., Nonlinear, Soft Matter Phys., 2012, 85, 051131.

51 V. Kharchenko and I. Goychuk, New J. Phys., 2012, 14, 043042.

52 I. Goychuk and V. Kharchenko, Math. Modell. Nat. Phenom., 2013, 8, 144.

53 V. Kharchenko and I. Goychuk, Phys. Rev. E: Stat., Nonlinear, Soft Matter Phys., 2013, 87, 052119.

54 Yu. A. Makhnovskii, V. M. Rozenbaum, D.-Y. Yang, S. H. Lin and T. Y. Tsong, Phys. Rev. E: Stat., Nonlinear, Soft Matter Phys., 2004, 69, 021102.

55 I. Goychuk, V. Kharchenko and R. Metzler, PLoS One, 2014, 9, e91700.

56 K. B. Zeldovich, J.-F. Joanny and J. Prost, Eur. Phys. J. E: Soft Matter Biol. Phys., 2005, 17, 155.
57 H. Kojima, E. Muto, H. Higuchi and T. Yanagida, Biophys. J., 1996, 73, 2012.

58 T. G. Mason and D. A. Weitz, Phys. Rev. Lett., 1995, 74, 1250.

59 T. A. Waigh, Rep. Prog. Phys., 2005, 68, 685.

60 R. A. L. Jones, Soft Condensed Matter, Oxford University Press, Oxford, 2009.

61 A. Gemant, Physics, 1936, 7, 311.

62 J. C. Maxwell, Philos. Trans. R. Soc. London, 1867, 157, 49.

63 U. Weiss, Quantum Dissipative Systems, World Scientific, Singapore, 2nd edn, 1999.

64 L. Bruno, V. Levi, M. Brunstein and M. A. Desposito, Phys. Rev. E: Stat., Nonlinear, Soft Matter Phys., 2009, 80, 011912.

65 A. W. Harrison, D. A. Kenwright, T. A. Waigh, P. G. Woodman and V. J. Allan, Phys. Biol., 2013, 10, 036002.

66 R. G. Palmer, D. L. Stein, E. Abrahams and P. W. Anderson, Phys. Rev. Lett., 1984, 53, 958.

67 K. Sekimoto, J. Phys. Soc. Jpn., 1997, 66, 1234.

68 I. Derenyi, M. Bier and R. D. Astumian, Phys. Rev. Lett., 1999, 83, 903.

69 D. Suzuki and T. Munakata, Phys. Rev. E: Stat., Nonlinear, Soft Matter Phys., 2003, 68, 021906.

70 H. Wang and G. Oster, Europhys. Lett., 2002, 57, 134.

71 J. Wyman, Proc. Natl. Acad. Sci. U. S. A., 1975, 72, 3983.

72 T. C. Gard, Introduction to Stochastic Differential Equations, Dekker, New York, 1988.

73 W. Deng and E. Barkai, Phys. Rev. E: Stat., Nonlinear, Soft Matter Phys., 2009, 79, 011112.

74 J.-H. Jeon and R. Metzler, Phys. Rev. E: Stat., Nonlinear, Soft Matter Phys., 2012, 85, 021147.

75 B. Alberts, A. Johnson, J. Lewis, M. Raff, K. Roberts and P. Walter, Molecular Biology of the Cell, Garland Science, New York, 4th edn, 2002. 